\title{
Concentration of electrostatic solitary waves around magnetic nulls within magnetic reconnection diffusion region: single-event-based statistics
}

Shiyou $\mathrm{Li}^{1^{*}}$, Shifeng Zhang ${ }^{1}$, Hong $\mathrm{Cai}^{1}$ and Sufang $\mathrm{Yu}^{2,3}$

\begin{abstract}
It is important to study the 'concentrated' electrostatic solitary waves/structures (ESWs) associated with the magnetic reconnection. In the literature published as regards this topic, very few studies have reported the observation of such a large number of ESWs in a single magnetic reconnection event. In this work, we report our observation of a large number of ESWs around the magnetic null-pairs within the magnetic reconnection ion diffusion region of Earth's magnetosphere on 10 September 2001. With more than 9,600 cases of ESWs observed around magnetic null-pairs and more than 97,600 cases observed during the ion diffusion region crossing time span, the observation of such a large number of ESWs in the diffusion region has not been reported often in published works. We further perform single-event-based statistical analysis of the characteristics of the ESWs around magnetic null-pairs. Based on the statistical result, we speculate that the two-stream instability originating from the magnetic null and traveling outward along the plasma sheet boundary layer (PSBL) is the candidate mechanism of the large number of observed ESWs. Our observation and analysis in this work suggests that even with the presence of a complex magnetic structure around a magnetic null-pair in the three-dimensional regime, concentrated ESWs can be observed. This single-reconnection-event-based statistical result of ESWs around the magnetic null-pairs can aid in understanding the microdynamics associated with three-dimensional (3D) magnetic reconnection.
\end{abstract}

Keywords: Magnetic reconnection; Magnetic null; Electrostatic solitary wave

\section{Background}

The observation of electrostatic solitary waves (ESWs) and the study of their structure within the magnetic reconnection diffusion region of Earth's magnetosphere has attracted a surge of research interest recently (Matsumoto et al. 2003; Deng et al. 2004, 2009; Cattell et al. 2005; Li et al. 2009, 2010, 2012, 2013a, 2013b, 2014). ESWs were first observed in the plasma sheet boundary layer (PSBL) in the magnetotail by Matsumoto et al. (1994). Thereafter, they have been widely observed in many key regions in space, including the PSBL in the magnetotail (Matsumoto et al. 1994, 1999; Kojima et al. 1999; Cattell et al. 1999), the auroral and cusp regions

\footnotetext{
* Correspondence: li.shiyou.qiu@gmail.com

${ }^{1}$ College of Aerospace Science and Engineering, National University of Defense Technology, Changsha 410073, China

Full list of author information is available at the end of the article
}

(Mozer et al. 1997; Cattell et al. 1999, Pickett et al. 2004), and the terrestrial bow-shock, magnetosheath, and magnetopause regions (e.g., Bale et al. 1998; Cattell et al. 2002, 2003; Matsumoto et al. 2003; Pickett et al. 2003, 2005; Shin et al. 2008; Li et al. 2013c).

Magnetic reconnection is the most important mechanism for the energy transformation from magnetic energy to kinetic energy and its transportation from the source region to other regions of interest. Both the structure and the microphysics related to reconnection have been extensively studied. As a primary focus of these studies, the ESWs associated with magnetic reconnection have also been investigated recently both through in situ observations (Deng et al. 2004, 2009; Cattell et al. 2005; Li et al. 2009, 2010, 2013b) and simulations (Drake et al. 2003; Cattell et al. 2005). Fujimoto and Machida (2006) studied the generation mechanism of ESWs on the PSBL 
associated with magnetic reconnection by using 2-1/2-D kinetic simulations in a large system. Some types of ESWs have been observed in the vicinity of the magnetic reconnection diffusion region on the dayside (Matsumoto et al. 2003) and in the magnetotail (Deng et al. 2004; Cattell et al. 2005). Recently, certain statistical studies have been performed on the characteristics and structure of the ESWs associated with magnetic reconnection (Li et al. 2009, 2013b).

However, in previous publications, the ESWs are only regarded as one kind of plasma wave and the number of reported ESWs is fairly limited. For example, Deng et al. (2009) reported several ESWs around the magnetic null in only one case. Li et al. (2009) found only several hundreds of such cases within several tens of magnetic reconnection events. It is important to study the 'concentrated' ESWs within the magnetic reconnection diffusion region for purely study on the ESWs associated with magnetic reconnection. We have found an event of magnetic reconnection with a large number of ESWs only after surveying more than 20 cases of reconnection events during the tail season of the Cluster orbit. In the present study, we examine this unique event of magnetic reconnection in the near-Earth magnetotail that is rich with ESWs (approximately 123,000), which was detected by the Cluster Wideband (WBD) plasma wave receiver (Gurnett et al. 1997) onboard the Cluster spacecraft SC1, SC3, and SC4.

\section{Methods}

The magnetic reconnection that occurred in the nearEarth magnetotail at approximately $-19 R_{E}$ on 10 September 2001 has been studied by Wang et al. (2008, 2010) extensively. They mainly focused on the energetic electrons within the magnetic reconnection ion diffusion region (Wang et al. 2008) and the energetic particle distribution in different subareas within this region (Wang et al. 2010). The crossing of all the spacecrafts to the ion diffusion region during the time interval of 07:50 to 08:05 UT has been confirmed by key observation characteristics by Wang et al. $(2008,2010)$ which characteristics include the following: (1) A reversal of high-speed flow $V_{x}$ (from negative to positive) coinciding with a reversal of $B_{z}$ (from negative to positive), and (2) observation of an out-ofplane Hall magnetic field $B_{y}$ by the four satellites of the Cluster spacecrafts. Most recently, Viberg et al. (2013) studied the plasma waves within this ion diffusion region and they identified three kinds of wave that were all observed within the separatrix regions: Langmuir waves (LWs), ESWs, and electron cyclotron waves (ECWs). Significantly, ECWs were observed for the first time within the magnetic reconnection diffusion region.

This event provides a great opportunity for us to perform statistical studies on the characteristics of the ESWs within the reconnection diffusion region around the magnetic null-pair. In this work, single-event-based statistical analysis of the characteristics of the ESWs around magnetic null-pairs is performed. The amplitudes, pulse widths, and the spatial scale of the ESWs both in the region adjacent to the magnetic null points and those observed during the entire diffusion-regioncrossing time span are analyzed in detail.

\section{Results and discussion}

\section{D magnetic reconnection event overview}

To provide an overview of this magnetic reconnection event, we plot the $\mathrm{X}$-component of the plasma velocity $\left(V_{x}\right)$ and the magnetic field $\left(B_{x}\right)$ from 07:55 UT to 07:59 $\mathrm{UT}$ in the upper panels of Figure 1. All data are presented in geocentric solar- magnetospheric (GSM) coordinates. The plasma data have been obtained from the Cluster ion spectrometry (CIS) experiment (Réme et al. 1997) with spin-time resolution (4 s) while the magnetic field data have been obtained from the fluxgate magnetometer (FGM) (Balogh et al. 1997) with full-time resolution (approximately $22.45 \mathrm{~Hz}$ ). In the bottom panels of Figure 1, we plot the Poincaré indices and the corresponding value of $\nabla \cdot B$, which are calculated by applying the method introduced by Greene (1992) and developed by Zhao et al. (2005), within the time interval from 07:55:00 UT to 07:59:00 UT. The Poincaré-index method has been successfully employed to infer the presence of a true magnetic null point (Priest and Titov 1996; Xiao et al. 2006, 2007; He et al. 2008a, 2008b; Deng et al. 2009).

The crossing of all the spacecrafts to the ion diffusion region during the time interval of 07:50 to 08:00 UT has been confirmed by means of certain key observation characteristics in the study by Wang et al. $(2008,2010)$. Wang et al. $(2008,2010)$ observed the four spacecrafts directly crossing the ion diffusion region, with $\mathrm{C} 1, \mathrm{C} 2$, and $\mathrm{C} 4$ crossing mainly from the northern side of the current layer $\left(B_{x}>0\right)$ and $C 3$ from the southern side $\left(B_{x}<0\right.$, refer to Figure 1a). We suggest that the spacecrafts skimmed the magnetic ion diffusion region along the boundary layer during the plasma flow reversal interval.

It is known that, when the Poincare index values +1 or -1 , there may be a magnetic null that lies within the Cluster tetrahedron (Xiao et al. 2006, 2007). The corresponding $\nabla \cdot B$ value (Figure $1 \mathrm{~d}$ ) is very small $(\nabla \cdot B \rightarrow 0$ and lies between -0.005 to 0.005 , and these values are indicated by the two horizontal dotted lines), thereby confirming that the singular points are physical magnetic nulls which lie within the Cluster tetrahedron (Cai et al. 2001; Xiao et al. 2006, 2007). The magnetic nulls indicated by the Poincaré index as shown in Figure 1a are observed nearly within the time span over which the plasma flow reverses (Figure 1b). The types of null, i.e., A-type, B-type, As-type, and Bs-type, are determined by 


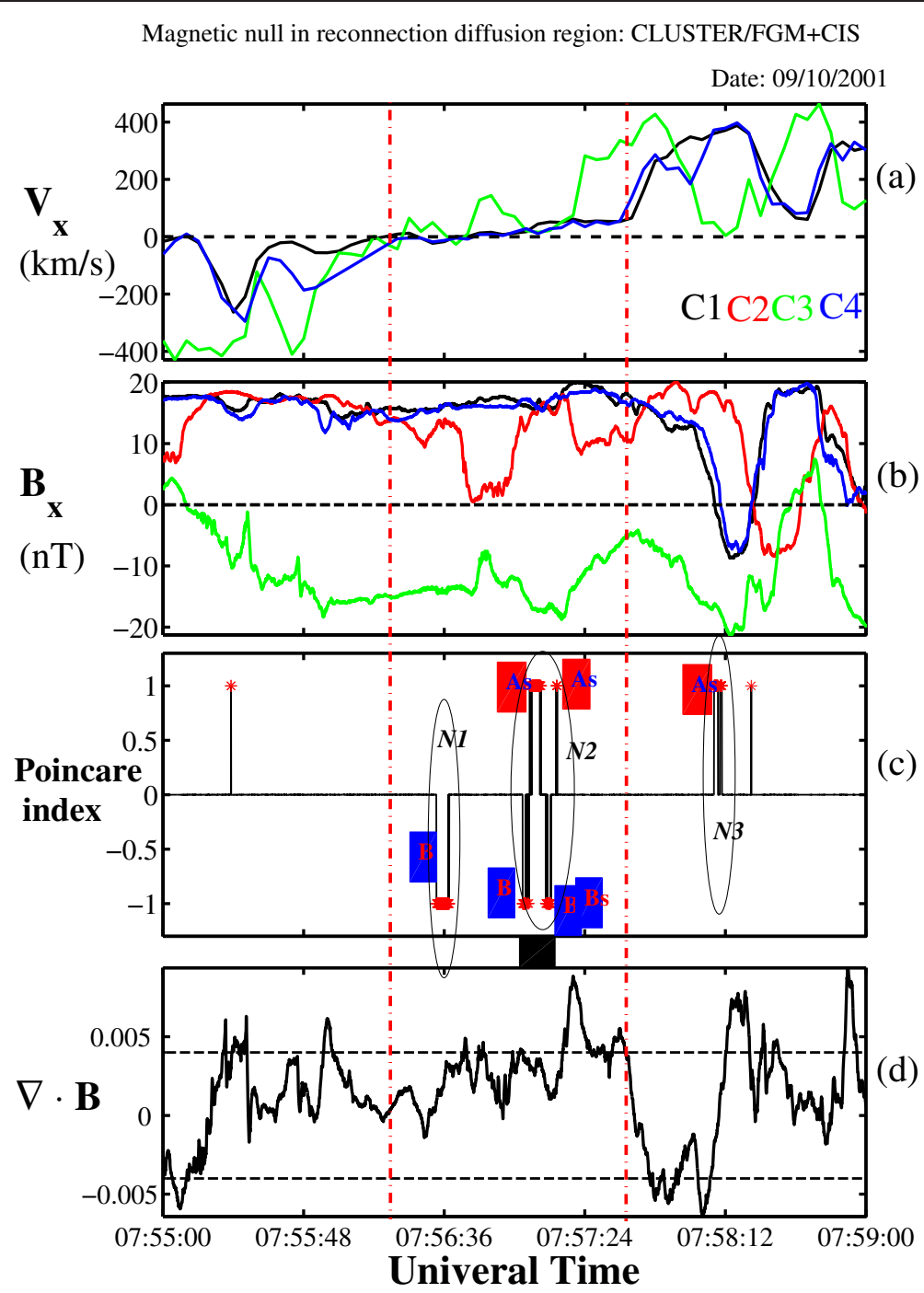

Figure 1 Overview of 3D magnetic reconnection on 10 September 2001. All data are shown within the time interval of 07:55 to 07:59 UT. In each panel, black, red, green, and blue lines represent $\mathrm{C} 1, \mathrm{C}_{2}, \mathrm{C} 3$, and C4 observations, respectively. (a) X-components of the plasma (proton) velocity observed by Cluster ion spectrometry (CIS). (b) X-component of magnetic field with data resolution of $22.4 \mathrm{~Hz}$. (c, d) Poincaré index and the corresponding $\nabla \cdot B$ values. The three ellipses denote the three groups of magnetic nulls or null-pairs. The flow reversal occurs at approximately 07:56:24 to 07:57:36 UT, and this interval is marked by two vertical dashed lines. The black bar between panels (c) and (d) denotes the time interval in Figure 2.

the eigenvalues of the $\delta \boldsymbol{B}$ matrix, which is a $3 \times 3$ real matrix, where $\delta \boldsymbol{B}=\partial \boldsymbol{B}_{i} / \partial \boldsymbol{x}_{j}$ (Priest and Titov, 1996), of which the eigenvalues are listed in the last row of Table 1. The A-type and As-type nulls are classified as positive nulls because the Poincaré index is +1 and the corresponding eigenvalue of $\delta \boldsymbol{B}$ matrix is positive. The field lines along the spine line of the A-type null direct outward from the null, and the field lines on the fan plane direct toward the null. The field lines around the As-type null exhibit a spiral structure that is more complex than that is observed in the A-type null (He et al. 2008b; Deng et al. 2009). The B-type and Bs-type nulls are classified as negative nulls since the Poincaré index is -1 , and the field line direction around these types of null is opposite those of the A-type or As-type null.

Table 1 lists the detailed characteristics of the magnetic nulls within the plasma flow reversal interval. They can be divided into three groups according to the observation time: N1 (07:56:33.3 to 07:56:37.8 UT), N2 (07:57:02.7 to $07: 57: 14.6 \mathrm{UT})$, and N3 (07:58:08.0 to 07:58:10.8 UT), which are denoted by the three ellipses in Figure 1c. Therefore, the type of magnetic null can be identified based on the characteristics for these three groups. Point N1 corresponds to the B-type magnetic null and N3 corresponds to the As-type magnetic null. N2 contains several magnetic nulls that coupled together 
Table 1 Characteristics of the magnetic null and null-pair within the reconnection diffusion region

\begin{tabular}{|c|c|c|c|c|c|c|c|}
\hline & \multirow[t]{2}{*}{ N1 } & \multicolumn{5}{|l|}{ N2 } & \multirow[t]{2}{*}{ N3 } \\
\hline & & $\mathrm{N} 2-\mathrm{a}$ & N2-b & N2-C & N2-c & N2-d & \\
\hline Time interval & $\begin{array}{l}07: 56: 33.419 \text { to } \\
07: 56: 37.746\end{array}$ & $\begin{array}{l}07: 57: 2.811 \text { to } \\
07: 57: 4.952\end{array}$ & $\begin{array}{l}07: 57: 5.219 \text { to } \\
07: 57: 9.099\end{array}$ & $\begin{array}{l}07: 57: 10.705 \text { to } \\
07: 57: 11.552\end{array}$ & $\begin{array}{l}07: 57: 11.597 \text { to } \\
07: 57: 12.489\end{array}$ & $\begin{array}{l}07: 57: 14.273 \text { to } \\
07: 57: 14.585\end{array}$ & $\begin{array}{l}07: 58: 8.06 \text { tc } \\
07: 58: 10.736\end{array}$ \\
\hline $\begin{array}{l}\text { Representative } \\
\text { time point }\end{array}$ & 07:56:33.955 & 07:57:03.435 & 07:57:9.099 & 07:57:11.418 & 07:57:12.043 & 07:57:14.407 & 07:58:10.736 \\
\hline Null type & B & B & As & B & Bs & As & As \\
\hline$\nabla \cdot B$ & 0.0016 & 0.0037 & 0.0031 & 0.0019 & 0.0013 & 0.0019 & 0.0019 \\
\hline$|\nabla \times B|$ & 0.0982 & 0.1926 & 0.1472 & 0.0908 & 0.0605 & 0.0845 & 0.105 \\
\hline$|\nabla \cdot B /| \nabla \times B \mid$ & $1.63 \%$ & $1.92 \%$ & $2.11 \%$ & $2.09 \%$ & $2.15 \%$ & $2.25 \%$ & $1.81 \%$ \\
\hline \multirow[t]{3}{*}{ Eigenvalue $\lambda_{1}$} & +0.0011 & +0.0022 & $\begin{array}{l}+0.0004+ \\
0.0016 i\end{array}$ & +0.0019 & $+0.0024+0.0036 i$ & $+0.0007+0.0013 i$ & $\begin{array}{l}-0.0012+ \\
0.0034 i\end{array}$ \\
\hline & +0.0063 & +0.0051 & $\begin{array}{l}+0.0004- \\
0.0016 i\end{array}$ & +0.0036 & $+0.0024-0.0036 i$ & $+0.0007-0.0013 i$ & $\begin{array}{l}-0.0012- \\
0.0034 i\end{array}$ \\
\hline & -0.0058 & -0.0036 & +0.0024 & -0.0036 & -0.0035 & +0.0006 & +0.0042 \\
\hline
\end{tabular}

to form a B-As-B(Bs)-As structure, which is regarded as a coupled magnetic null-pair because of the small angle of about $3^{\circ}$ to $7^{\circ}$ between the $\gamma$-line of one null and the $\sum$-surface of the other null for each null-pair. Herein, the $\gamma$-line represents a virtual line along the magnetic field emerging from the magnetic null and the $\sum$-surface represents a virtual surface where the magnetic field with opposite orientations on its two sides (Lau and Finn 1990; Priest and Titov 1996; Xiao et al. 2006, 2007).

The magnetic null point and null-pair form the crucial regions of a three-dimensional (3D) magnetic reconnection, wherein the magnetic field lines break and then reconnect. Investigation of the detailed structures, waves, and particle dynamics around the magnetic null points is significantly important for understanding the reconnection in the $3 \mathrm{D}$ regime. The spatial structure of the magnetic null or null-pairs will be studied in another work.

\section{Observation of ESWs}

The primary observations presented here are mainly obtained from the WBD plasma wave receiver (Gurnett et al. 1997) onboard the Cluster spacecrafts. The WBD performs only 1D measurements along one axis only within the spin plane of the spacecraft (Pickett et al. 2005). Moreover, the WBD provides continuous observations of the electric field waveforms with a time resolution of $9.5 \mathrm{kHz}$. As regards the 3D magnetic reconnection event in question, the WBD instruments onboard SC1, $\mathrm{SC} 3$, and SC4 work well in the $9.5-\mathrm{kHz}$ bandwidth mode, thereby providing the best opportunity for the study of ESWs associated with magnetic reconnection in the near-Earth tail. In the present work, we examined the $9.5-\mathrm{kHz}$ bandwidth mode of the WBD data carefully, and we observed a large number of ESWs continuously forming along the trajectory of all the four spacecrafts around the magnetic null-pairs and within the ion diffusion region.

We firstly surveyed the WBD data of Cluster when the magnetic null and null-pairs, i.e., N1 (07:56:33.3 to $07: 56: 37.8 \mathrm{UT})$ and N2 (07:57:02.7 to 07:57:14.6 UT), were detected during the plasma flow reversal. Numerous ESWs were observed within these two time intervals. Figure 2 shows the observations of the WBD instruments within the second time interval (07:57:00 to 07:57:12 UT), during which the magnetic null-pairs were detected and were involved by the Cluster tetrahedron. The WBD waveforms $\left(E_{z}\right)$ and the corresponding spectrograms are shown for SC1 (panels a and b), SC3 (panels c and d), and SC4 (panels e and $\mathrm{f}$ ). The local electron plasma frequency $\left(f_{p e}\right)$ and cyclotron frequency $\left(f_{c e}\right)$ are superposed in red and black lines, respectively. It is worth noting that $E_{z}$ is one of the measurements of the electric field made by the WBD instrument (another is $E_{y}$ ) in the spin plane of the spacecraft, which is nearly parallel to the ambient magnetic field in this region. As shown, the waves are enhanced mainly over a wide range in the frequency domain (several hundred to several thousand hertz, i.e., $f_{c e} \sim f_{p e}$ ) Herein, $f_{c e}=336 \mathrm{~Hz}$ for a magnetic strength of $12 \mathrm{nT}$ and $f_{p e}=5.692 \mathrm{kHz}$ for an electron density of $0.4 \mathrm{~cm}^{-3}$. The frequency range for the wave enhancement is consistent with the signature of broadband electrostatic noise (BEN) (Matsumoto et al. 1994). The spike-shaped waveforms support the speculation that this kind of BEN results from ESWs. The continuous observation of the spiked solitary wave structures suggests that ESWs can be observed around the magnetic null-pair in the diffusion region.

Figure 3 displays the typical waveforms of the ESWs observed by Cluster/SC1 around the first group of magnetic null-pairs which are shown in Figure 1c. Figure 3a displays the waveforms of $E_{z}$ within a time span of slightly more than $1 \mathrm{~s}$ starting at 07:56:32.4290 UT, and 


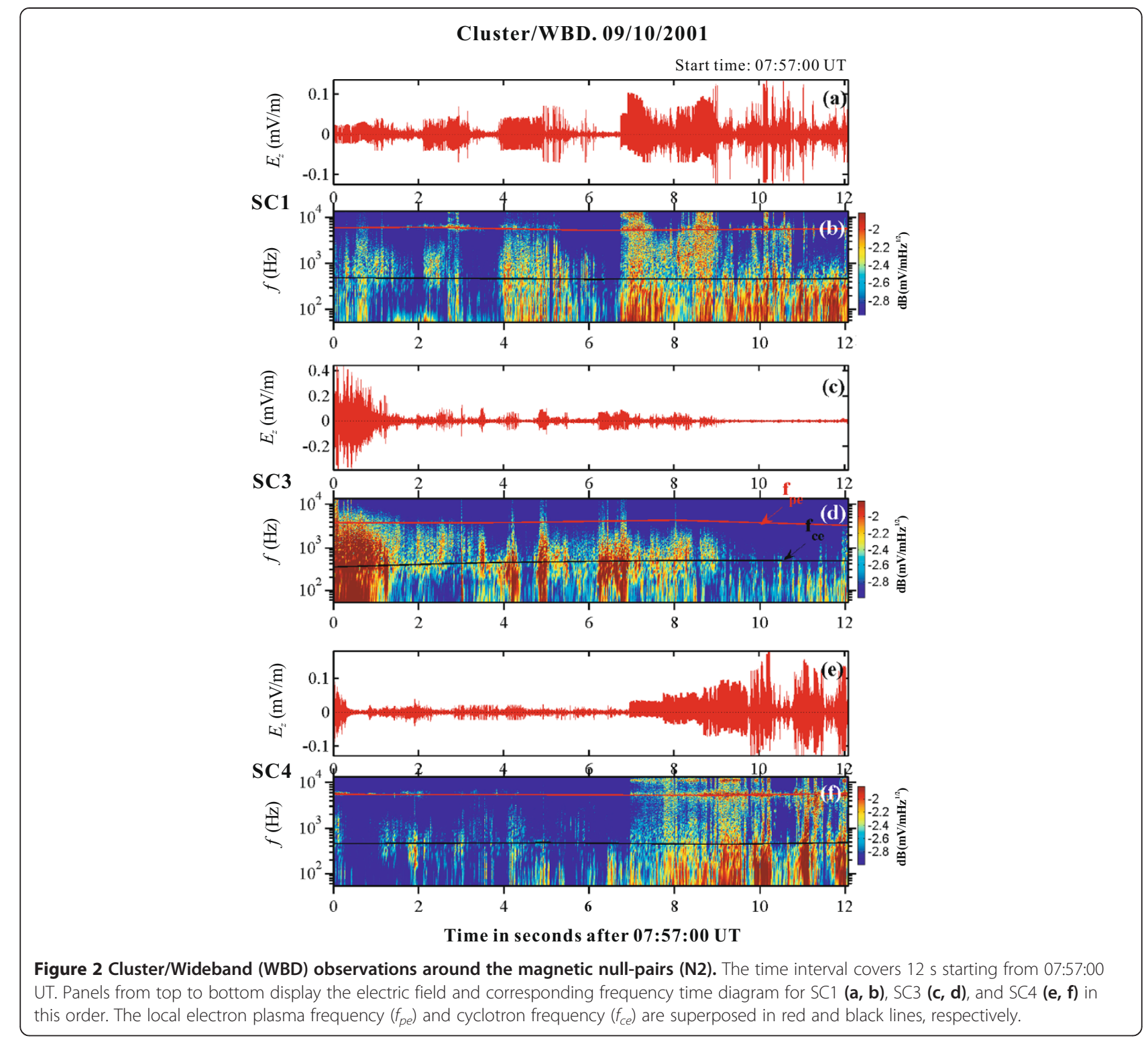

Figure $3 \mathrm{~b}$ shows the magnified waveform over a span of approximately $40 \mathrm{~ms}$, corresponding to the black bar (07:56:32.429 to 07:56:32.469 UT) in Figure 3a, in order to highlight the details of the waveform. The waveform comprises clear spike-shaped solitary nonlinear pulses. These waveforms are typical ESWs. Both bipolar and tripolar pulses ( $\mathrm{Li}$ et al. 2013b) can be seen clearly in these ESW waveforms. Because the tripolar ESWs for this case study constitute only a small portion of all the observed ESWs, we do no analyze such waveforms.

In the magnetic reconnection case in question, numerous ESWs were observed. Since the number of ESWs is very large, the automatic waveform selection (AWS) method introduced by Kojima et al. (2000) was employed to extract the ESW waveforms and to collect their parameters. We also surveyed the WBD data within the second time interval around the second group of magnetic nullpairs. Similarly, numerical ESWs were observed. From the WBD data, we determined that approximately 12,100 ESW waveforms were observed within the time interval of 07:56:00 to 07:57:30 UT during which the magnetic nullpairs were detected by the Cluster tetrahedron. Upon considering the entire reconnection diffusion crossing span from 07:50 to 08:00 UT, an enormous number of ESWs $(123,200$, i.e., 10 times of that observed in the adjacent magnetic null-pair) could be detected. The reporting of such a large number of ESWs that were continuously observed within one reconnection diffusion region is rare in published works.

As to the basic characteristics of the ESWs, we define the four following four aspects to perform statistical analysis. (1) Type of ESWs (Li et al. 2014): there are two 


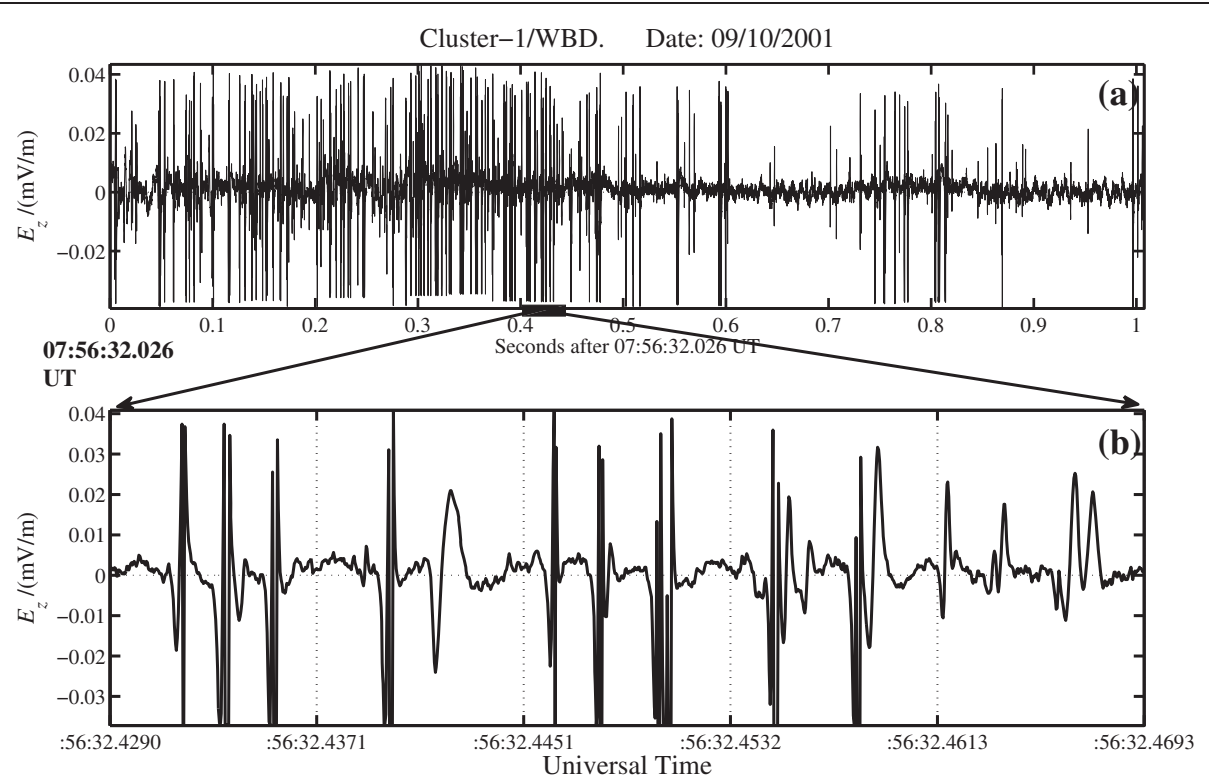

Figure 3 Electric field observed by Cluster-1/Wideband (WBD). (a) 1 second waveform after 07:56:32.026 UT. (b) Waveforms zoomed-in within time interval of 07:56:32.4290 UT to 07:56:32.4693 UT. Waveforms exhibiting spiked solitary structures are shown within the observational time period of magnetic null points (group 1). Clear bipolar and tripolar electrostatic solitary waves (ESWs) are seen.

types of ESWs with respect to bipolar waveforms. One type comprises negative-to-positive bipolar solitary structures and the other type comprises positive-tonegative bipolar waveforms. These two types of ESWs are designated as 'type-A-ESW' (negative-to-positive) and 'type-B-ESW' (positive-to-negative) for convenience by
Li et al. (2014). (2) 'Solitariness' of the ESW waveform: coupled ESWs and isolated ESWs. The coupled ESW comprises a coupled waveform whose pulse interval is smaller than their individual pulse widths (refer to Figure 4a). (3) ESWs with and without offset fields (refer to Figure 4b). (4) ESWs with asymmetrical and symmetrical waveforms
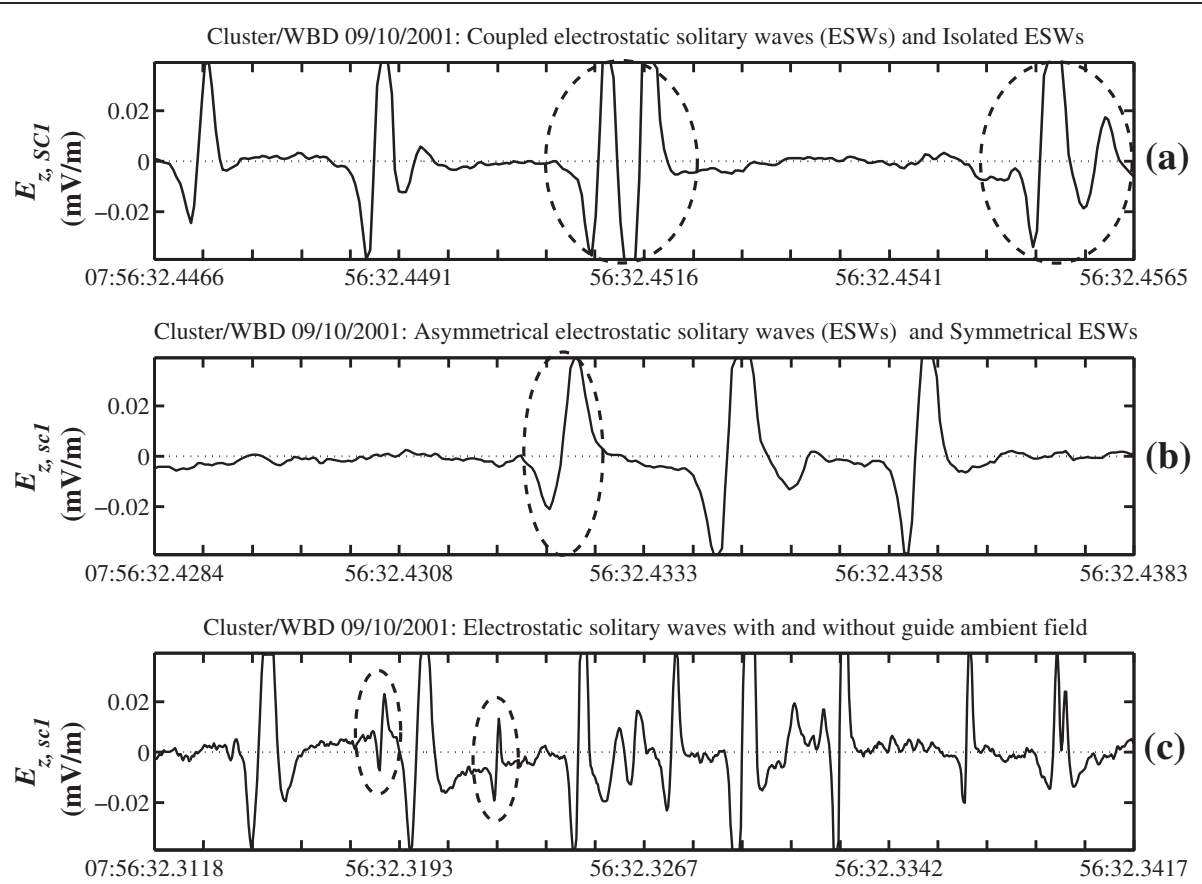

Figure 4 Typical series of electrostatic solitary waves (ESWs) observed by SC1 at 07:56:32 UT. The solitary pulses indicated by the dashed eclipses in panels (a-c) represent the coupled ESWs, the ESWs with offset field, and the asymmetrical ESWs in that order. 
(refer to Figure 4c). Figure 4 presents several typical series of ESWs observed by SC1 at 07:56:32 UT, which are all 'type-B-ESW'. Figure 4a depicts sample coupled ESWs and isolated ESWs, wherein the interval between the two spiked pulses marked by the dashed ellipse is considerably smaller than the pulse width of the isolated ESW pulse. We defined two indexes, symmetrical index $I_{\text {sym }}=\mid E_{\max }-$ $E_{\text {aver }}|/| E_{\text {min }}-E_{\text {aver }} \mid$ and the guide index $I_{\text {guide }}=\left|E_{\text {aver }}\right| / \mid$ $E_{\max }-E_{\text {min }} \mid$, where $E_{\max }$ and $E_{\text {min }}$ denote the maximum and minimum amplitudes of the solitary pulse, respectively, and $E_{\text {aver }}$ represents the averaged value. For a solitary ESW pulse, when $I_{\text {guide }}>0.1$, the pulse can be regarded as an ESW with an offset field, and when $0.9<$ $E_{\text {sym }}<1.1$, the pulse can be viewed as a symmetrical ESW. Figure $4 \mathrm{c}$ depicts the plots of several waveforms comprising asymmetrical and symmetrical ESWs, wherein the solitary pulse marked by a dashed ellipse represents an asymmetrical ESW. Figure 4b depicts ESWs with and without offset fields, wherein the two small solitary pulses in the dashed ellipse denote ESWs with an offset field. We note that the first spiked wave in Figure 4c depicts a tripolar ESW (Li et al. 2013b). Since the occurrence rate of tripolar ESWs is generally considerably smaller than that of bipolar ESWs, we neglect tripolar ESWs in the present work as regards our statistical analysis.

Primitive statistics is performed on the ESWs obtained by $\mathrm{SC} 1, \mathrm{SC} 3$, and $\mathrm{SC} 4$ within two time intervals. The first time interval corresponds to the duration over which the spacecrafts are around the magnetic null-pairs (group 1), and the second corresponds to the time spent within the entire magnetic reconnection diffusion region (group 2). The statistical information concerning the detected ESWs is summarized and listed in Table 2. The total number of ESWs in group 1 is 12,111 while it is 123,291 (about 10 times that in group 1) in group 2. The table provides a comparison of the ESWs with respect to the following characteristics: 1) type of ESWs: type-B ESW and type-A ESW; 2) solitariness of the waveform of ESWs: coupled ESWs and isolated ESWs; 3) ESWs with and without offset field; and 4) asymmetrical and symmetrical ESWs. This information concerning the ESWs within the magnetic reconnection ion diffusion region and around the magnetic null-pairs as summarized in Table 2 suggests that most of the ESWs exhibit a solitary, nonlinear evolution structure; however, a small number of the observed ESWs exhibit coupled waveforms, thereby suggesting that the electron holes are in the development stage when two adjacent electron holes merge. It is interesting that most of the solitary structures are observed with an offset electric field, which may reflect some type of lowfrequency turbulence or background DC electric field. The ESWs have perfect symmetrical structure for the most part, thereby suggesting the presence of symmetrical electron potential holes in this region.
Table 2 Summary of the statistical information of electrostatic solitary waves (ESWs)

\begin{tabular}{lll}
\hline & Group 1 & Group 2 \\
\hline Total & 12,111 & 123,291 \\
$\begin{array}{l}\text { Type } \\
\text { Type-A }\end{array}$ & \\
Type-B & $5,968(49.3 \%)$ & $61,871(50.2 \%)$ \\
Solitariness & $6,143(50.7 \%)$ & $61,420(49.8 \%)$ \\
Coupled & & \\
Solitary & $1,200(9.9 \%)$ & $14,063(11.4 \%)$ \\
If offset & $10,911(90.1 \%)$ & $109,228(88.6 \%)$ \\
Offset & & \\
$\quad$ Not offset & $7,683(63.4 \%)$ & $80,033(64.9 \%)$ \\
Symmetry & $4,428(36.6 \%)$ & $43,258(35.1 \%)$ \\
Asymmetrical & & $37,268(30.2 \%)$ \\
Symmetrical & $3,736(30.8 \%)$ & $86,023(69.8 \%)$ \\
\hline
\end{tabular}

Observed near the magnetic nulls within the magnetic reconnection diffusion region on 10 September 2001.

Group 1: Data corresponding to the ESWs observed during 07:56:30 to 07:57:30 UT (close to the nulls); Group 2: Data corresponding to the ESWs observed during 07:50 to 08:00 UT (the entire diffusion region crossing).

\section{Statistical analysis of ESW characteristics}

The observation of such a large number of ESWs within a single reconnection diffusion region presents evidence of wave/turbulence activities during the ongoing magnetic reconnection process, and this presents a good opportunity for us to understand such waves via a statistical study. In the following sections, we perform statistical analysis of the characteristics of the ESWs observed within the magnetic reconnection diffusion region.

\section{Amplitude and pulse width of ESWs within diffusion region in $V_{x}-B_{x}$ plane}

The magnetic structure around a 3D magnetic null or null-pair appears as an 2D X-type configuration viewed from proper viewing angles (He et al. 2008a), e.g., in a diagonal direction in the X-Y plane in He et al. (2008a). As mentioned previously, a large number of ESWs were observed in the vicinity of the magnetic null on both the northern side and the southern side of the X-line when viewed using a 2D model (He et al. 2008a). In this subsection, we discuss our statistical analysis of the peak-to-peak amplitudes and the pulse widths of the ESWs associated with the magnetic reconnection diffusion region in the $V_{x}-B_{x}$ plane, which roughly represents the diffusion region in the magnetotail in the $\mathrm{X}-\mathrm{Z}$ plane.

The results of our statistical analysis are shown in Figure 5. The top row displays the results for the ESWs observed during the interval of 07:56:30 to 07:57:30 UT (near the magnetic null-pair), and the bottom row corresponds to those observed during the entire diffusion region crossing. Panels (a) and (c) show the results 

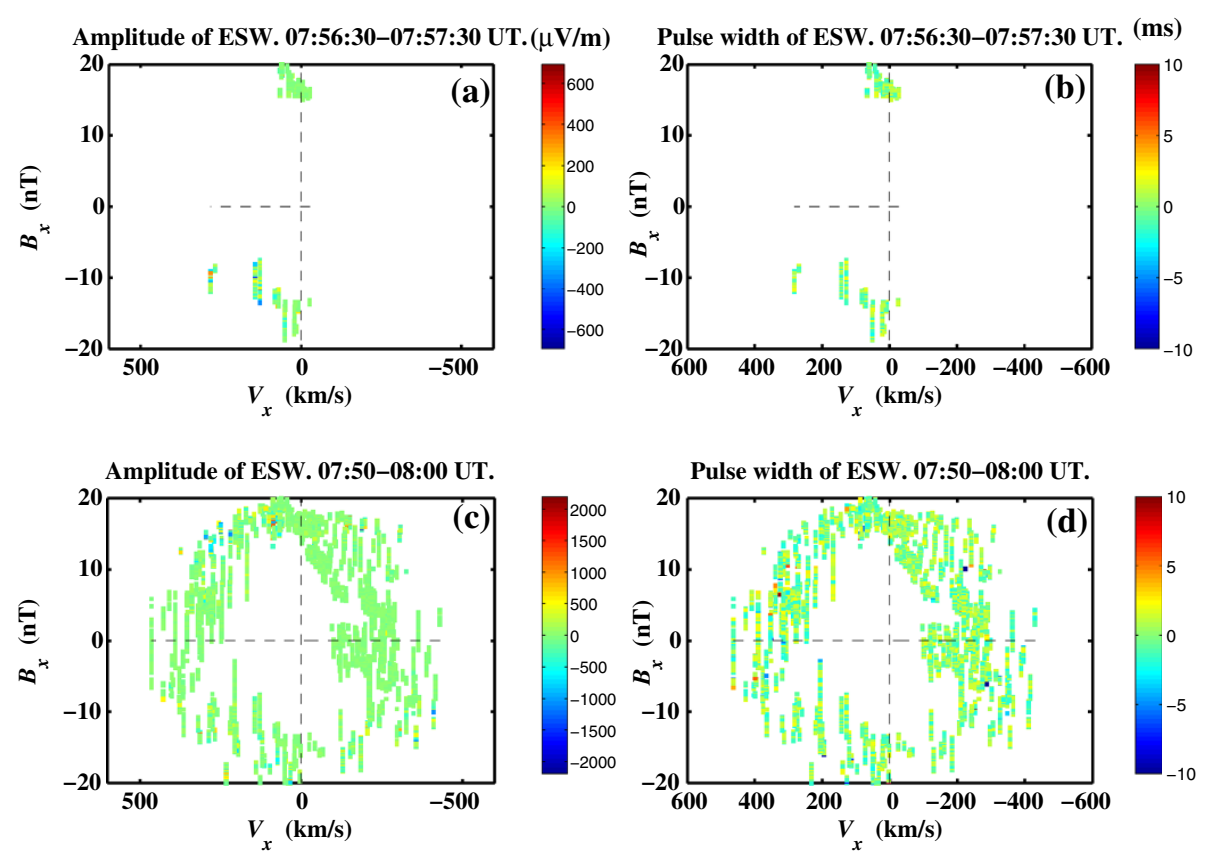

Pulse width of ESW. 07:50-08:00 UT.

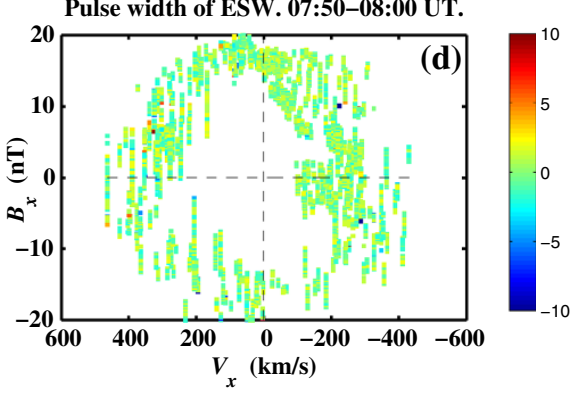

Figure 5 Statistical results for the amplitudes and pulse widths of ESWs. Panels $(\mathbf{a}, \mathbf{b})$ are for the ESWs observed in the PSBL around magnetic nulls and panels $(\mathbf{c}, \mathbf{d})$ are for those observed within the entire reconnection diffusion region on 10 September 2001.

concerning the ESW amplitudes, and panels (b) and (d) show the results for the pulse widths. It is to be noted that the magnitude of the amplitude or pulse width is color coded, wherein positive values represent type-B ESWs (negative-to-positive) and the negative values represent type-A ESWs (positive-to-negative). The results indicate that type-A and type-B ESWs are 'interleaved' within the diffusion region represented by the $V_{x}-B_{x}$ plane.

As regards the ESW amplitudes, our analysis shows that, during this magnetic reconnection process, the amplitudes of the observed ESWs in the vicinity of the X-line (magnetic null-pairs) are mainly less than $1 \mathrm{mV} / \mathrm{m}$ (ranging from approximately $0.01 \mathrm{mV} / \mathrm{m}$ to $0.7 \mathrm{mV} / \mathrm{m}$ ), while for the whole data set of the ESWs within the ion diffusion region, the amplitude varies over a wider range from approximately $0.01 \mathrm{mV} / \mathrm{m}$ to approximately $2 \mathrm{mV} / \mathrm{m}$.

Bench tests with the WBD flight spare instrument were recently carried out at Iowa. In the tests, some solitary waves in the form of bipolar and tripolar pulses were input using a signal generator, and the output was examined in terms of the pulse shape and duration.
These tests show that any pulses of these types that are observed in the WBD data and that have time durations longer than the suggested guidelines should not be used for analysis purposes other than in this case that the researcher clearly states that these pulses, although considered to be solitary waves, cannot be characterized in terms of specific shape, time duration, or amplitude (refer to: Swanner et al, http://www-pw.physics.uiowa.edu/cluster/ pulse_tests.pdf). Thus, the ESWs observed near the magnetic null points that have pulse widths greater than $2 \mathrm{~ms}$ are not suitable for analysis of shape, amplitudes, and time durations.

In the present study, the solitary pulses were analyzed regardless of their pulse. We examined the data set that was studied, and we found that ESWs with pulse widths of less than $2 \mathrm{~ms}$ dominate the observations. This result is listed in Table 3 in detail. For those ESWs observed adjacent to the magnetic null (07:56:30 to 07:57:30 UT), $79.4 \%$ of the ESWs have pulse widths of less than $2 \mathrm{~ms}$; while within the whole time span (07:50 to 08:00 UT), this ratio is equal to $79.2 \%$. Thus, we eliminate those

Table 3 Pulse widths of ESWs observed within the reconnection diffusion region on 10 September 2001

\begin{tabular}{|c|c|c|c|c|c|c|c|}
\hline Time span & & Total & $<1 \mathrm{~ms}$ & $<2 \mathrm{~ms}$ & $<3 \mathrm{~ms}$ & $<4 \mathrm{~ms}$ & $<5 \mathrm{~ms}$ \\
\hline \multirow[t]{2}{*}{$07: 56: 30$ to $07: 57: 30$} & Number & 12,111 & 5,097 & 9,613 & 10,711 & 10,979 & 11,407 \\
\hline & Ratio & $100 \%$ & $42.0857 \%$ & $79.3741 \%$ & $88.4403 \%$ & $90.6531 \%$ & $94.1871 \%$ \\
\hline \multirow[t]{2}{*}{ 07:50:00 to 08:00:00 } & Number & 123,291 & 48,560 & 97,641 & 109,618 & 112,238 & 116,153 \\
\hline & Ratio & $100 \%$ & $39.3865 \%$ & $79.1956 \%$ & $88.9100 \%$ & $91.0350 \%$ & $94.2104 \%$ \\
\hline
\end{tabular}


ESWs with pulse widths larger than $2 \mathrm{~ms}$ for the statistical study. The numbers of the ESWs that are valid for analysis are 9,613 and 97,641 for group 1 and group 2, respectively.

\section{Spatial scale estimation}

The ESWs in space are regarded to as electron phasespace potential holes (electron holes, EHs) that are the Bernstein-Greene-Kruskal (BGK) modes, i.e., 1D equilibrium solutions to the time-independent Vlasov-Poisson equations [Bernstein et al. 1957; Chen and Parks 2002]. In this section, we replace 'ESWs' with 'EHs' on studying the spatial scale of the corresponding spatial structure of the ESWs. The spatial scale of the EHs observed in nature is usually estimated by assuming the propagating speed of the waves along the magnetic field or by calculating the speed through interferometer measurements (Franz et al. 1998) from single-satellite observations. Though multiple-satellite analysis presents another approach to study the spatial scale, in this subsection, we only estimate the spatial scale of ESWs in space by considering the propagation of ESWs along the ambient magnetic field. We remark here because the 3D structure of ESWs cannot be constructed by the WBD onboard Cluster, we simply take into account the scale of the ESWs parallel to the ambient magnetic field.

The propagation speed of ESWs is obtained by calculating the time lags between different spacecraft, which are on the same magnetic flux tube and observing the same ESWs, and the distance in the direction along the magnetic field tube between these satellites. However, the direct evidence of ESW propagation in the present event is not available because of the large distance between the spacecrafts. Thus, we consider the electron beam velocity for the estimation of the spatial scale of EHs. In general, the propagating speed of the ESWs is related to the electron beam velocity if the ESWs are formed during the electron beam instability. Though the electron beam velocity is different in different regions, as a single-event-based statistical study, it is feasible to assume that the ESWs can propagate with a speed in a fixed range during one reconnection process. The spatial scale of the ESWs is estimated by assuming that the ESWs are propagating outward of the X-line along the PSBL with a velocity of $1,500 \mathrm{~km} / \mathrm{s}$. This velocity is close to the velocity assumed by Kojima et al. (1999) for those ESWs observed in the tail lobe and the tail PSBL regions. This value is also consistent with the results obtained from the Cluster multi-satellite analysis carried out by Pickett et al. (2004) in the auroral region and near the dayside magnetopause.

Figure 6 shows the statistical results of the estimated spatial scales of the ESWs based on the observations of a single magnetic reconnection diffusion region crossing on 10 September 2001. The panels in left column correspond to the ESWs observed between 07:56:30 UT
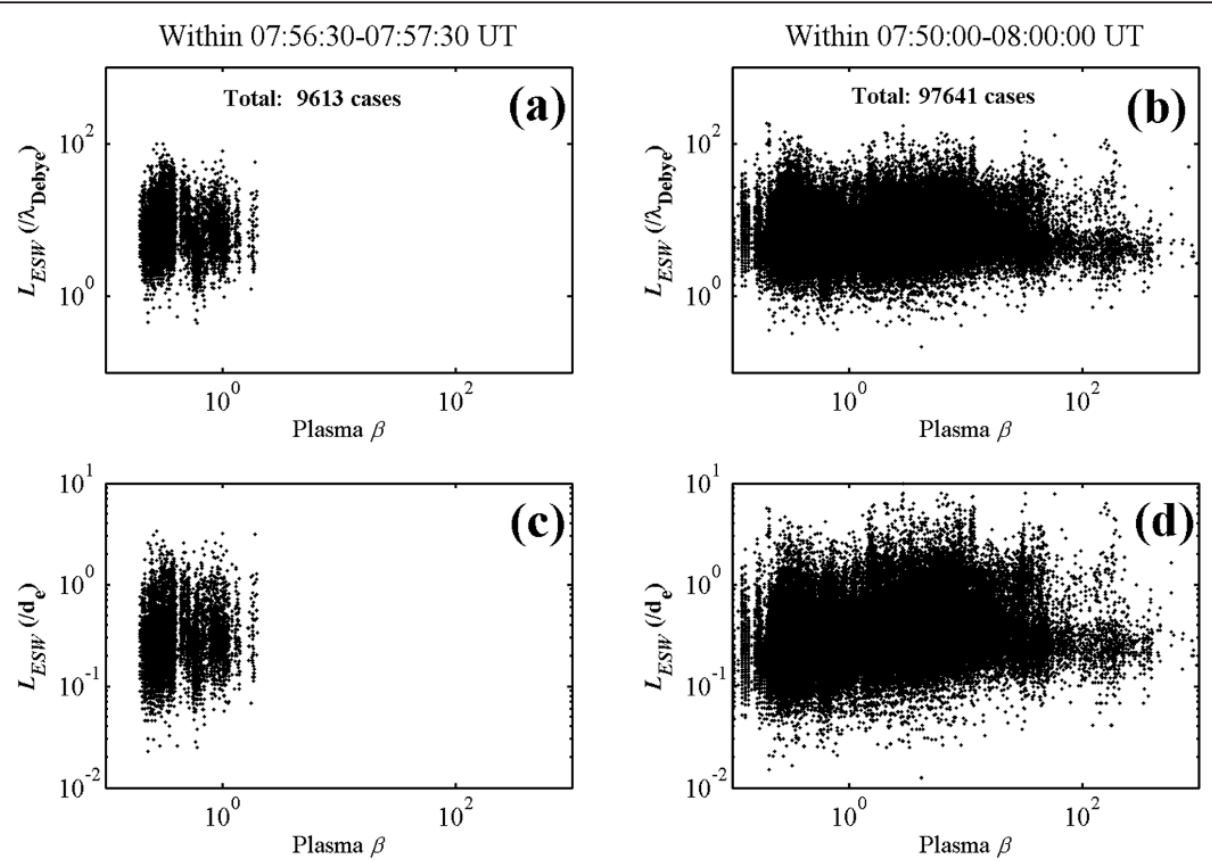

Figure 6 Statistical results of the estimated spatial scale of ESWs. Panels (a) and (c) on the left column are For electrostatic solitary waves (ESWs) observed near the magnetic null-pairs (group 1 ESWs); Panels (b) and (d) on the right column are for ESWs observed during the entire reconnection diffusion region crossing time period (group 2 ESWs). The top $(\mathbf{a}, \mathbf{b})$ and bottom panels $(\mathbf{c}, \mathbf{d})$ in each column show the scale normalized by the Debye length $\left(\lambda_{D}\right)$ and electron skin length $\left(d_{e}\right)$, respectively. 
and 07:57:30 UT (hereafter referred to as group $1 \mathrm{ESWs}$ ) when Cluster was located in the PSBL near the magnetic null-pair while the right panels correspond to those observed over a wider time span of 07:50 to 08:00 UT (hereafter referred to as group 2 ESWs) during which the spacecrafts skimmed through the entire diffusion region. The spatial scale of the ESWs is normalized respectively by Debye length $\left(\lambda_{D}\right)$ in the upper row and by the electron inertial length $d_{e}=c / \omega_{p e}$ (i.e., the electron skin depth) in the lower row.

To better show the results for the spatial scale, we provide a scatter plot of this scale as a function of plasma $\beta$, which can be an indicator of the location of the spacecraft (and the ESWs) relative to the PSBL. As has been shown, the locations of the Cluster spacecraft were mainly in the PSBL, and therefore, the ESWs observed in this case are mainly propagating in the PSBL. Thus plasma $\beta$, which is plotted on the horizontal axis in each panel, ranges mainly between 0.1 and 1 for group 1 ESWs. However, group 2 ESWs, because the spacecraft are in an orbit close to the neutral current sheet, the plasma $\beta$ can have a considerably large ratio of $\beta>1$, thereby suggesting that the ESWs are observed on the PSBL side of the reconnection ion diffusion region.

The width of the ESWs is related to the Debye length. In the early days, a statistical study of the spatial scales of solitary pulses (i.e., ESWs) by FAST satellite observations (Ergun et al. 1998, 1999) in the auroral ionosphere revealed that solitary pulses with a positive potential typically have a Gaussian half-width ranging from less than one $\lambda_{D}$ to several $\lambda_{D}$ with a mean value of $1.80 \lambda_{D}$ and a standard deviation of $1.13 \lambda_{D}$. The WIND satellite also observed bipolar, Debye-scale electrostatic structures in the transition region of the Earth's collisionless bow shock (Bale et al. 2002). The latest observation of electron phase-space holes (EHs), i.e., ESWs, in Earth's plasma sheet by the THEMIS satellites reveals that the ESWs are found to have a spatial scale of tens of Debye lengths along the ambient magnetic field (Andersson et al. 2009). Figure 6a,b clearly shows that most of the ESWs observed by the Cluster spacecraft around the magnetic nulls within the reconnection diffusion region have a spatial scale between 10 and $100 \lambda_{D}$. This statistical result for scale length is slightly larger than that observed in the auroral ionosphere (Ergun et al. 1998, 1999) and in the shock transition region (Bale et al. 2002) but consistent with that observed by THEMIS in Earth's plasma sheet (Andersson et al. 2009).

The statistical results in Figure $6 \mathrm{c}, \mathrm{d}$ shows that the group 1 ESWs lie in the range between 0.05 and $1 d_{e}$ for the most part, while for group 2 ESWs, the scale range is wider, 0.01 to $5 d_{e}$. Though a statistical effect maybe exists, i.e., it is possible to result in a wider range of the spatial scale of ESWs in statistics since the group 2
ESWs number about 10 times that of group 1 ESWs; however, this statistical result can still suggest that the farther away the ESW is from the magnetic reconnection null (or X-line), the larger it is expected to be as a solitary structure. Moreover, the ESW scale is mainly comparable with the electron inertial scale, thereby suggesting that the electron dynamics is the main evolving process (e.g., nonlinear evolution of the electron two-stream instabilities) in the formation and propagation of the ESWs as revealed by simulation results (e.g., Omura et al. 1996; Fujimoto and Machida 2006).

\section{Discussion}

Most recently, Viberg et al. (2013) have studied the same reconnection event and the same WBD dataset in great detail. They have presented detailed observations of electrostatic HF waves and related electron distributions within the reconnection diffusion region for the same event. Moreover, they have mapped the observations of different wave types to different parts within this region, and they have proposed possible generation mechanisms. However, in our study, we are mainly concerned with the overall characteristics of ESWs that are concentrated in one reconnection diffusion region and may dominate the waves. Such a concentration of ESWs suggests that the ESWs may play a key role in energy release during the reconnection process.

Recent 2-1/2-D particle-in-cell kinetic simulation results have suggested that the electrostatic turbulence plays an important role in strong electron energization in the plasma sheet-lobe boundary region during magnetic reconnection (Fujimoto and Machida 2006). The statistics concerning the single magnetic reconnection reveal that the ESWs are mainly observed on the PSBL side of the reconnection ion diffusion region. In this event, the spacecraft detected numerous ESWs (group 1 ESWs) when plasma flow reversal was detected by Cluster, i.e., near the magnetic null observation region, thus suggesting that the PSBL near reconnection null may be an important region that is responsible for the generation of ESWs. Fujimoto and Machida (2006) also suggest that the two-stream instability in the PSBL includes an electron beam with highand low-energy electrons. The former originates from the lobe region in the vicinity of the reconnection X-line (or magnetic null), while the latter originates from the opposite boundary region of the plasma sheet. We suggest that the two-stream instability originated from the magnetic null and its outward release along the PSBL is the candidate generation mechanism of the large number of ESWs observed in our study.

\section{Conclusions}

In the present work, by examining the $9.5-\mathrm{kHz}$ bandwidth mode of the WBD data carefully, we determined 
that a large number ESWs was continuously observed along the trajectory of all the four spacecrafts around the magnetic null-pairs (more than 9,600 valid observations) and within the ion diffusion region (more than 97,600 valid observations). Such a large number of ESWs has not often been observed in the diffusion region as per previously published works.

Primitive statistics was performed for an overview of these sets of hundreds of thousands of ESWs around the magnetic null-pairs and within the ion diffusion region. Our results showed that most of the ESWs exhibit a solitary, nonlinear evolution structure; most of the solitary structures are observed with an offset electric field, which may reflect some kind of low-frequency turbulence. The ESWs exhibit a perfectly symmetrical structure for the most part, thereby indicating the presence of symmetrical electron potential holes in space near the magnetic nulls and within the ion diffusion region.

With such a large number of ESWs being observed within a single magnetic reconnection, the amplitudes, pulse widths, and the spatial scale of the ESWs are analyzed in detail. The main conclusions of these statistics can be summarized as follows:

(1)The ESWs are mainly observed in the plasma sheet boundary layer of the reconnection region, but a small number of ESWs is observed in the region near the magnetic null-pairs within the diffusion region. The amplitude of ESWs near the magnetic null-pairs is smaller than those in the region farther away from the reconnection X-line or the magnetic null-pairs.

(2)Most of the ESWs have pulse widths of the order of $<2 \mathrm{~ms}$, both for the ESWs near the magnetic null (79.4\%) and within a wider spatial range in the diffusion region (79.2\%).

(3) Most of the ESWs observed by the Cluster spacecraft around the magnetic nulls within the reconnection diffusion region have a spatial scale between 10 and $100 \lambda_{D}$, which result is consistent with that observed by THEMIS in Earth's plasma sheet (Andersson et al. 2009).

(4) The ESW scale is mainly comparable with the electron inertial scale, thereby suggesting that the electron dynamics is the main evolving process. The farther away is the ESW from the magnetic reconnection null (or X-line), the larger it is expected to be as a solitary structure.

(5)Our observation and analysis in this work suggests that concentrated ESWs can be observed even with the existence of a complex magnetic structure around the magnetic null-pair in the three-dimensional regime.
Very few studies have reported on ESWs in such high concentrations observed in such a region near the magnetic null-pair, which is similar to the X-line in the traditional 2D region considered for magnetic reconnection. In our next work, we plan to perform detailed statistical studies of the characteristics of ESWs within the magnetic reconnection diffusion region based on this set of ESWs. A computer particle simulation is necessary for examining their generation mechanism and their roles within the magnetic reconnection diffusion region.

\section{Abbreviations}

BEN: broadband electrostatic noise; ECWs: electron cyclotron waves; ESWs: electrostatic solitary waves; LW: Langmuir waves; PSBL: plasma sheet boundary layer; WBD: wideband plasma wave receiver.

\section{Competing interests}

Numerous ESWs are observed around the magnetic null-pairs within the magnetic reconnection ion diffusion region during one single ion diffusion region crossing process on 10 September 2001. It is rather rare in the published works. Primitive single-event-based statistical study is performed. The ESWs have a spatial scale between 10 and $100 \lambda D$, and they are mainly comparable with the electron inertial scale, thereby suggesting that the electron dynamics is the main evolving process. Such a concentrating set of ESWs suggests that the ESWs would be of key role in energy releasing during reconnection process.

\section{Authors' contributions}

LSY and ZSF conceived the study. LSY performed most of data analysis and manuscript composition. ZSF and CH helped LSY do the analysis and helped in drafting the manuscript. YSF helped LSY analyze part of the data. All authors read and approved the final manuscript.

\section{Acknowledgements}

The authors acknowledge the efforts of all the members of the Cluster team for obtaining the high-quality data. This work has made use of the Cluster Active Archive (CAA). S. Y. Li thanks J. S. Pickett for providing the WBD data and for her helpful discussion. This work is supported by the National Natural Science Foundation of China under Grant No. 41304132, the Post-Doctoral Research Program of China (42956), the 53-class General Financial Grant from the China Post-Doctoral Science Foundation under Grant No. 2013 M532115, and the National Basic Research Program of China.

\section{Author details}

'College of Aerospace Science and Engineering, National University of Defense Technology, Changsha 410073, China. ${ }^{2}$ College of Forestry, Guangxi University, Nanning 530005, China. ${ }^{3}$ College of Forest Resources and Environment, Nanjing Forestry University, Nanjing 21003, China.

Received: 5 January 2014 Accepted: 21 November 2014

Published online: 31 December 2014

\section{References}

Andersson L, Ergun RE, Tao J, Roux A, Lecontel O, Angelopoulos V, Bonnell J, McFadden JP, Larson DE, Eriksson S, Johansson T, Cully CM, Newman DL, Goldman MV, Glassmeier KH, Baumjohann W (2009) New features of electron phase space holes observed by the Themis Mission. Phys Rev Lett 102:225004, doi:10.1103/PhysRevLett.102.225004

Bale SD, Kellogg PJ, Larson DE, Lin RP, Goetz K, Lepping RP (1998) Bipolar electrostatic structures in the shock transition region: evidence of electron phase space holes. Geophys Res Lett 25:2929-2932

Bale SD, Hull A, Larson DE, Lin RP, Muschietti L, Kellogg PJ, Goetz K, Monson SJ (2002) Electrostatic turbulence and Debye-scale structures associated with electron thermalization at collisionless shocks. Astrophys J 575:L25-L28

Balogh A, Dunlop MW, Cowley SWH, Southwood DJ, Thomlinson JG, Glassmeier KH, Musmann G, Lühr H, Buchert S, Acuña MH, Fairfield DH (1997) The Cluster magnetic field investigation. Space Sci Rev 79:65

Bernstein IB, Greene JM, Kruskal MD (1957) Exact nonlinear plasma oscillations. Phys Rev Lett 108:546-550 
Cai DS, Li YT, Ichikawai T, Xiao C, Nishikawa K (2001) Visualization and criticality of magnetotail field topology in a three-dimensional particle simulation. Earth Planets Space 53:1011

Cattell C, Dombeck J, Wygant J, Hudson MK, Mozer FS, Temerin MA, Peterson WK (1999) Comparisons of Polar satellite observations of solitary wave velocities in the plasma sheet boundary and the high altitude cusp to those in the auroral zone. Geophys Res Lett 26:425-428

Cattell CA, Crumley J, Dombeck J, Wygant JR, Mozer FS (2002) Polar observations of solitary waves at the Earth's magnetopause. Geophys Res Lett 29(5):1065, doi:10.1029/2001GL014046

Cattell C, Neiman C, Dombeck J, Crumley J, Wygant J, Kletzing CA, Peterson WK, Mozer FS (2003) Large amplitude solitary waves in and near the Earth's magnetosphere, magnetopause and bow shock: Polar and Cluster observations. Nonlinear Proc Geophys 10:13

Cattell C, Dombeck J, Wygant J, Drake JF, Swisdak M, Goldstein ML, Keith W, Fazakerley A, André M, Lucek E, Balogh A (2005) Cluster observations of electron holes in association with magnetotail reconnection and comparison to simulations. J Geophys Res 110, A01211, doi:10.1029/2004JA010519

Chen LJ, Parks GK (2002) BGK electron solitary waves in 3D magnetized plasma. Geophys Res Lett 29(9):1331, doi:10.1029/2001GL013385

Deng XH, Matsumoto H, Kojima H, Mukai T, Anderson RR, Baumjohann W, Nakamura R (2004) Geotail encounter with reconnection diffusion region in the Earth's magnetotail: evidence of multiple $X$ lines collisionless reconnection? J Geophys Res 109, A05206, doi:10.1029/2003JA010031

Deng XH, Zhou M, Li SY, Baumjohann W, Andre M, Cornilleau N, Santolík O, Pontin Dl, Reme H (2009) Dynamics and waves near multiple magnetic null points in reconnection diffusion region. J Geophys Res 114, A07216, doi:10.1029/2008JA013197

Drake JF, Swisdak M, Cattell C, Shay MA, Rogers BN, Zeiler A (2003) Formation of electron holes and particle energization during magnetic reconnection. Science 299:873-877, doi:10.1126/science.1080333

Ergun RE, Carlson CW, McFadden JP, Mozer FS, Roth I, Strangeway RJ (1998) Debye-scale plasma structures associated with magnetic-field-aligned electric fields. Phys Rev Lett 81:826-829, doi:10.1103/PhysRevLett.81.826

Ergun RE, Carlson CW, Muschietti L, Roth I, McFadden JP (1999) Properties of fast solitary structures. Nonlinear Proc Geophys 6:187-194

Franz JR, Kintner PM, Pickett JS (1998) Polar observations of coherent electric field structures. Geophys Res Lett 25:1277-1280, doi:10.1029/98GL50870

Fujimoto K, Machida S (2006) A generation mechanism of electrostatic waves and subsequent electron heating in the plasma sheet-lobe boundary region during magnetic reconnection. J Geophys Res 111, A09216, doi:10.1029/2005JA011542

Greene JM (1992) Locating three-dimensional roots by a bisection method. J Comput Phys 98:194-198

Gurnett DA, Huff RL, Kirchner DL (1997) The wide-band plasma wave investigation. Space Sci Rev 79:195-208

He JS, Tu CY, Tian H, Xiao CJ, Wang XG, Pu ZY, Ma ZW (2008a) A magnetic null geometry reconstructed from Cluster spacecraft observations. J Geophys Res 113, A05205, doi:10.1029/2007JA012609

He JS, Zong QG, Deng XH, Tu CY, Xiao CJ, Wang XG, Ma ZW (2008b) Electron trapping around a magnetic null. Geophys Res Lett 35, L14104, doi:10.1029/2008GL034085

Kojima H, Omura Y, Matsumoto H, Miyaguti K, Mukai T (1999) Characteristics of electrostatic solitary waves observed in the plasma sheet boundary: statistical analyses. Nonlinear Proc Geophys 6:179-186

Kojima H, Omura Y, Matsumoto H, Miyaguti K, Mukai T (2000) Automatic waveform selection method for electrostatic solitary waves. Earth Planets Space 52:495-502

Lau YT, Finn JM (1990) Three-dimensional kinematic reconnection in the presence of field nulls and closed field lines. Astrophys J 350:672-691

Li SY, Deng XH, Zhou M, Tang RX, Liu K, Kojima H, Matsumoto H (2009) Statistical study of electrostatic solitary waves associated with reconnection: Geotail observations. J Adv Space Res 43(3):394-400, doi:10.1016/j.asr.2008.05.013

Li SY, Deng XH, Zhou M, Yuan ZG, Wang JF, Lin X, Lin MH, Fu S (2010) Cluster observation of electrostatic solitary waves around magnetic null point in thin current sheet. Chin Phys Lett 27(1):019401

Li SY, Zhang SF, Deng XH, Cai H (2012) Spatial evolution of electrostatic solitary waves along plasma sheet boundary layer adjacent to the magnetic reconnection X-line. Chin Phys Lett 29(8):089402
Li SY, Zhang SF, Deng XH, Cai H (2013a) Large bipolar signature in a perpendicular electric field of two-dimensional electrostatic solitary waves associated with magnetic reconnection: statistics and discussion. Chin Phys Lett 30(1):019401

Li SY, Zhang SF, Cai H, Chen XQ, Deng XH (2013b) Statistics of the tripolar electrostatic solitary waves within magnetic reconnection diffusion region in the near-Earth magnetotail. J Geophys Res 118:2911-2918, doi:10.1002/jgra.50209

Li SY, Zhang SF, Cai H, Deng XH, Chen XQ, Zhou M, Yang HB (2013c) Large 3-dimensional ellipsoid-sphere-shaped structure of electrostatic solitary waves in the terrestrial bow-shock under condition of $\Omega_{c e} / \omega_{p e} \ll 1$. Geophys Res Lett 40:3356-3361, doi:10.1002/grl.50623

Li SY, Omura Y, Lembège B, Deng H, Kojima H, Saito Y, Zhang SF (2014) Geotail observation of counter directed ESWs associated with the separatrix of magnetic reconnection in the near-Earth magnetotail. J Geophys Res Space Physics 119:202-210, doi:10.1002/2013JA018920

Matsumoto H, Kojima H, Miyatake T, Omura Y, Okada M, Nagano I, Tsutsui M (1994) Electrostatic solitary waves (ESW) in the magnetotail: BEN waveforms observed by Geotail. Geophys Res Lett 21:2915-2918, doi:10.1029/94GL01284

Matsumoto H, Frank LA, Omura Y, Kojima H, Paterson WR, Tsutsui M, Anderson RR (1999) Generation mechanism of ESW based on the GEOTAIL plasma wave observation, plasma observation and particle simulation. Geophys Res Lett 26:421-424

Matsumoto H, Deng XH, Kojima H, Anderson RR (2003) Observation of electrostatic solitary waves associated with reconnection on the dayside magnetopause boundary. Geophys Res Lett 30(6):1326, doi:10.1029/2002GL016319

Mozer FS, Ergun R, Temerin M, Cattell C, J. Dombeck J, Wygant J (1997) New features in time domain electric field structures in the auroral acceleration region. Phys Rev Lett 79(7):1281

Omura Y, Matsumoto H, Miyake T, Kojima H (1996) Electron beam instabilities as generation mechanism of electrostatic solitary waves in the magnetotail. J Geophys Res 101:2685-2697

Pickett JS, Menietti JD, Gurnett DA, Tsurutani B, Kintner PM, Klatt E (2003) Solitary potential structures observed in the magnetosheath by the Cluster spacecraft. Nonlinear Proc Geophys 10:3-11

Pickett JS, Kahler SW, Chen LJ, Huff RL, Santolík O, Khotyaintsev Y (2004) Solitary waves observed in the auroral zone: the Cluster multi-spacecraft perspective. Nonlin Proc Geophys 11:183-196

Pickett JS, Chen LJ, Kahler SW, Santolík O, Goldstein ML, Lavraud B (2005) On the generation of solitary waves observed by Cluster in the near-Earth magnetosheath. Nonlinear Proc Geophys 12:181-193

Priest ER, Titov VS (1996) Magnetic reconnection at three-dimensional null points. Phil Trans R Soc Lond A 354:2951-2992

Réme H, Bosqued JM, Sauvaud JA, Cros A, Dandouras J, Aoustin C, Bouyssou J, Camus T, Cuvilo J, Martz C, Medale JL, Perrier H, Romefort H, Rouzaud J, Uston CD, Mobius E, Crocker K, Granoff M, Kistler LM, Popecki M, Hovestadt D, Klecker B, Paschmann G, Scholer M, Carlson CW, Curtis DW, Lin RP, Mcfadden JP, Formisano V, Amata E, Bavassano-Cattaneo MB, Baldetti P, Belluci G, Bruno R, Chionchio G, Lellis AD, Shelley EG, Ghielmetti AG, Lennartsson W, Korth A, Rosenbauer H, Lundin R, Olsen S, Parks GK, Mccarthy M, Balsiger H (1997) The Cluster ion spectrometry (CIS) experiment. Space Sci Rev 79:303-350

Shin K, Kojima H, Matsumoto H, Mukai T (2008) Characteristics of electrostatic solitary waves in the Earth's foreshock region: Geotail observations. J Geophys Res 113:A03101b, doi:10.1029/2007JA012344

Swanner JM, Pickett JS, Phillips JR, Kirchner DL. WBD response to bipolar and tripolar pulses: bench tests vs. in flight observations. http://www-pw.physics. uiowa.edu/cluster/pulse_tests.pdf

Viberg H, Khotyaintsev W, Vaivads A, André M, Pickett JS (2013) Mapping HF waves in the reconnection diffusion region. Geophys Res Lett 40:1032-1037, doi:10.1002/grl.50227

Wang R, Lu Q, Guo J, Wang S (2008) Spatial distribution of energetic electrons during magnetic reconnection. Chin Phys Lett 25:3083-3085

Wang R, Lu Q, Li X, Huang C, Wang S (2010) Observations of energetic electrons up to $200 \mathrm{keV}$ associated with a secondary island near the center of an ion diffusion region: a Cluster case study. J Geophys Res 115, A11201, doi:10.1029/2010JA015473

Xiao CJ, Wang XG, Pu ZY, Zhao H, Wang JX, Ma ZW, Fu SY, Kivelson MG (2006) In situ evidence for the structure of the magnetic null in a 3D reconnection event in the Earth's magnetotail. Nature Phys 2:478-483, doi:10.1038/nphys342 
Xiao CJ, Wang XG, Pu ZY, Ma ZW, Zhao H, Zhou GP, Wang JX,

Kivelson MG (2007) Satellite observations of separator-line geometry of three-dimensional magnetic reconnection. Nature Physics 3:609-613, doi:10.1038/nphys650

Zhao H, Wang J, Zhang J, Xiao CJ (2005) A new method of identifying 3D null points in solar vector magnetic fields. Chin J Astron Astrophys 5:443-447

Submit your manuscript to a SpringerOpen ${ }^{\mathcal{D}}$ journal and benefit from:

- Convenient online submission

- Rigorous peer review

- Immediate publication on acceptance

- Open access: articles freely available online

- High visibility within the field

- Retaining the copyright to your article

Submit your next manuscript at $\gg$ springeropen.com 